Original article

\title{
A prospective study on the neurological complications of breast cancer and its treatment: Updated analysis three years after cancer diagnosis
}

\author{
Filipa Fontes a , Susana Pereira ${ }^{\text {a, b }}$, José Manuel Castro-Lopes ${ }^{c,}$, Nuno Lunet $^{\text {a, e, * }}$ \\ a ISPUP-EPIUnit, University of Porto (ISPUP), Rua das Taipas, $n^{\circ} 135,4050-600$, Porto, Portugal \\ ${ }^{\mathrm{b}}$ Portuguese Institute of Oncology of Porto, Rua Dr. António Bernardino de Almeida, 4200-075, Porto, Portugal \\ ${ }^{c}$ Department of Experimental Biology, Faculty of Medicine, University of Porto, Al. Prof. Hernâni Monteiro, 4200-319 Porto, Portugal \\ ${ }^{\mathrm{d}}$ Institute for Molecular and Cell Biology (IBMC), University of Porto, Rua do Campo Alegre, n'823, 4150-180 Porto, Portugal \\ e Department of Clinical Epidemiology, Predictive Medicine and Public Health, Faculty of Medicine, University of Porto, Al. Prof. Hernâni Monteiro, 4200-319, \\ Porto, Portugal
}

\section{A R T I C L E I N F O}

\section{Article history:}

Received 7 April 2016

Received in revised form

10 June 2016

Accepted 11 June 2016

Available online 7 July 2016

\section{Keywords:}

Breast neoplasms

Cognition disorders

Nervous system diseases

Neuropathic pain

Phantom limb

Polyneuropathies

\begin{abstract}
A B S T R A C T
Objectives: To quantify the prevalence of neurological complications among breast cancer patients at one and three years after diagnosis, and to identify factors associated with neuropathic pain (NP) and chemotherapy-induced peripheral neuropathy (CIPN).

Material and methods: Prospective cohort study including 475 patients with newly diagnosed breast cancer, recruited among those proposed for surgical treatment (Portuguese Institute of Oncology, Porto). Patients underwent a neurological evaluation and had their cognitive function assesses with the Montreal Cognitive Assessment, before treatment and at one and three years after enrollment. We estimated the prevalence of each neurological complication, and odds ratios (OR), adjusted for socio-demographic and clinical characteristics, to identify factors associated with NP and CIPN.

Results: More than half of the patients [54.7\%, 95\% confidence interval (95\%CI): 50.2-59.2] presented at least one neurological complication, at one or at three years after cancer diagnosis. Between the first and the third year of follow-up, there was an increase in the prevalence of NP (from 21.1\% to 23.6\%), cognitive impairment (from $7.2 \%$ to $8.2 \%$ ), cerebrovascular disease (from $0.6 \%$ to $1.5 \%$ ) and brain metastasis (from $0.0 \%$ to $0.6 \%$ ). The prevalence of CIPN decreased from $14.1 \%$ to $12.6 \%$. Axillary lymph node dissection was associated with NP at one year $(\mathrm{OR}=2.75,95 \% \mathrm{Cl}: 1.34-5.63)$ and chemotherapy with NP at three years $(\mathrm{OR}=2.10,95 \% \mathrm{CI}: 1.20-3.67)$. Taxane-based chemotherapy was strongly associated with prevalence of CIPN at one and three years.

Conclusion: Neurological complications are frequent even three years after cancer diagnosis and NP remained the major contributor to the burden of these conditions among survivors.
\end{abstract}

(C) 2016 Published by Elsevier Ltd.

\section{Introduction}

Breast cancer is the most frequent cancer among women, estimated to have accounted for approximately one quarter of all cases of cancer diagnosed in 2012 [1]. Access to early diagnosis through mammography screening and effective treatments [2] makes breast cancer one of those with a better prognosis. The 5-year net survival is now greater than $80 \%$ in most developed countries [3],

\footnotetext{
* Corresponding author. Department of Clinical Epidemiology, Predictive Medicine and Public Health, Faculty of Medicine, University of Porto, Al. Prof. Hernâni Monteiro, 4200-319 Porto, Portugal. Tel.: +351 225513652.

E-mail address: nlunet@med.up.pt (N. Lunet).
}

and this translates into a high number of women living for longer periods with possible sequelae of breast cancer and its treatment, emphasizing the relevance of a comprehensive study of the burden of cancer among survivors.

Neurological complications, either direct, namely metastatic disease, or due to indirect mechanisms, including vascular disorders, paraneoplastic syndromes or side-effects of treatments, may be a frequent source of morbidity among breast cancer patients $[4,5]$. We previously followed a cohort of breast cancer patients during the first year after diagnosis, and showed that nearly half of the women treated for breast cancer had at least one neurooncological complication and one quarter developed at least two during this period; the most frequent were neuropathic pain (NP) and chemotherapy-induced peripheral neuropathy (CIPN) [6]. 
The progression of cancer itself and the subsequent exposure to additional treatments, the late and/or cumulative effects of some options of cancer management, but also the possibility of recovering from some of the neuro-oncological complications over time, bring attention to the importance of a comprehensive assessment of the prevalence of these conditions in the long term. Therefore, we updated the follow-up of this cohort up to three years after diagnosis, aiming to quantify the prevalence of neurological complications among breast cancer patients, and to identify factors associated with NP and CIPN.

\section{Material and methods}

We conducted a prospective study with newly diagnosed breast cancer women, followed for three years. The study protocol has been described in detail elsewhere [7].

\section{Patients and setting}

Patients proposed for surgery were consecutively recruited in 2012, among those admitted to the Breast Clinic of the Portuguese Institute of Oncology of Porto, Portugal. We excluded women that had received any treatment for breast cancer before, those previously treated with chemotherapy and/or radiotherapy in the chest and/or axillary areas for other primary cancers, and those considered less likely to be able to cooperate due to cognitive impairment [score lower than 17 , or lower than 16 for women over 65 years, in the Montreal Cognitive Assessment (MoCA) [8,9]].

The cohort included 506 patients with incident breast cancer, from whom 31 were lost to follow-up until the three-year of followup (11 patients died, 10 abandoned the study, six could not be contacted, two were transferred to another hospital and two were considered unable to cooperate by the neurologist). Therefore, a total of 475 (93.9\%) completed the three-year follow-up evaluation with a median [percentile 25-percentile 75 (P25-P75)] time of follow-up of 1095 (1073-1126) days and were included in the present analysis. The patients lost to follow-up were not significantly different (participants vs. lost to follow-up) regarding age (median: 54.7 vs. 58.1 years, $\mathrm{p}=0.130$ ), education [median: 6 vs. 4 schooling years (4-6), $\mathrm{p}=0.081$ ] and cancer stage (stage $0 / \mathrm{I}: 53.9 \%$ vs. $48.4 \%, \mathrm{p}=0.581)$.

\section{Data collection}

All participants underwent a neurological evaluation at baseline (before any treatment) and at one and three years after enrollment.

Complementary exams (e.g.: computed tomography, magnetic resonance imaging, nerve conducting studies) were requested whenever indicated, according to the usual practice of the hospital. In all evaluations, socio-demographic data were collected using a structured questionnaire and clinical records were reviewed for cancer stage, breast cancer treatments and the presence of recurrence. Cancer stage was classified according to the American Joint Committee on Cancer staging manual [10].

\section{Prevalence of neurological complications}

Neurological complications affecting the patients at one and three years after cancer diagnosis were recorded; this included conditions identified de novo in any of these follow-up evaluations or diagnosed before, but still present at the follow-up evaluation.

CIPN was defined as peripheral neuropathy occurring after chemotherapy. Among subjects with peripheral neuropathy at baseline, CIPN was considered present only if there was a worsening of the preexisting neuropathy. The severity of CIPN was quantified using the Total Neuropathy Score, clinical version (TNSc) (range: 0 to 28) [11] and the Common Terminology Criteria for Adverse Events, V.4.0 (CTCAE) (range: 1 to 5) [12]. In both scales, higher scores represent greater severity.

NP was considered probable, according to the International Association for the Study of Pain (IASP) [13], if pain distribution was neuroanatomically plausible and history was suggestive of relevant lesions or diseases affecting the somatosensory system, plus negative or positive sensory signs in neurological examination, confined to the innervation territory of the injured nervous structure. Pain sensation and light touch sensation were assessed using a wood cocktail stick and a piece of cotton wool, respectively, as recommended by the IASP [13]. We considered NP secondary to breast cancer treatments as prevalent in each of the evaluations if it was present in the last $24 \mathrm{~h}$, in the breast, chest wall, axilla, or medial upper arm on the affected side, donor region of breast reconstruction, or in the hands/feet (secondary to CIPN). In order to quantify pain severity, the severity subscale of the Brief Pain Inventory Short Form was used [14]; it consists of a mean score of four questions measuring the worst, least, average and current pain in the past $24 \mathrm{~h}$ (range: 0 to 10 , with $0=$ "no pain" and $10=$ "pain as bad as you can imagine").

Among patients submitted to mastectomy, phantom breast syndrome was defined as the presence of the sensation that the removed breast is still present [15]. When in addition, patients described a sensation of pain in the removed breast, phantom breast pain was considered present [15] and the CTCAE was used to grade phantom pain (range: 1 to 5 ) [12].

Cognitive impairment was considered present when the patients' MoCA score (range: 0 to 30 ) was at least 2.0 standard deviations below age- and education-adjusted cut-offs for possible cognitive impairment [8].

\section{Statistical analysis}

Patients' characteristics were presented as counts and proportions for all categorical variables, and median and P25-P75 for quantitative variables.

Prevalence estimates and corresponding 95\% confidence intervals $(95 \% \mathrm{CI})$ were estimated for each of the neurological complications at one and three years after cancer diagnosis. The McNemar's test was used to compare the proportion of patients with each complication at one and three years.

Adjusted odds ratios (OR) and 95\%CI were computed using logistic regression, to quantify the relation between sociodemographic and clinical characteristics of the patients and the presence of NP and CIPN at one and three years after cancer diagnosis.

Statistical analyses were conducted using STATA ${ }^{\circledR}$, version 11.2 (StataCorp, College Station, TX, USA).

\section{Results}

Patients' characteristics

At baseline, half of the women had less than 55 years of age and more than two thirds had less than 10 years of education. A total of $6.5 \%$ were diagnosed with non-invasive breast cancer (ductal carcinoma in situ) and the remaining with invasive breast cancer stage I (47.4\%), II (30.7\%), III (14.7\%) or IV (0.6\%).

The breast cancer treatments performed during the first year after diagnosis are presented in Table 1. Nearly half of the patients were submitted to mastectomy and just over one third to axillary lymph node dissection (ALND). Most of the participants underwent adjuvant treatment. Docetaxel-based regimens were used by more than two thirds of women receiving chemotherapy. 
Table 1

Breast cancer treatments performed during the first year after diagnosis $(\mathrm{N}=475)$.

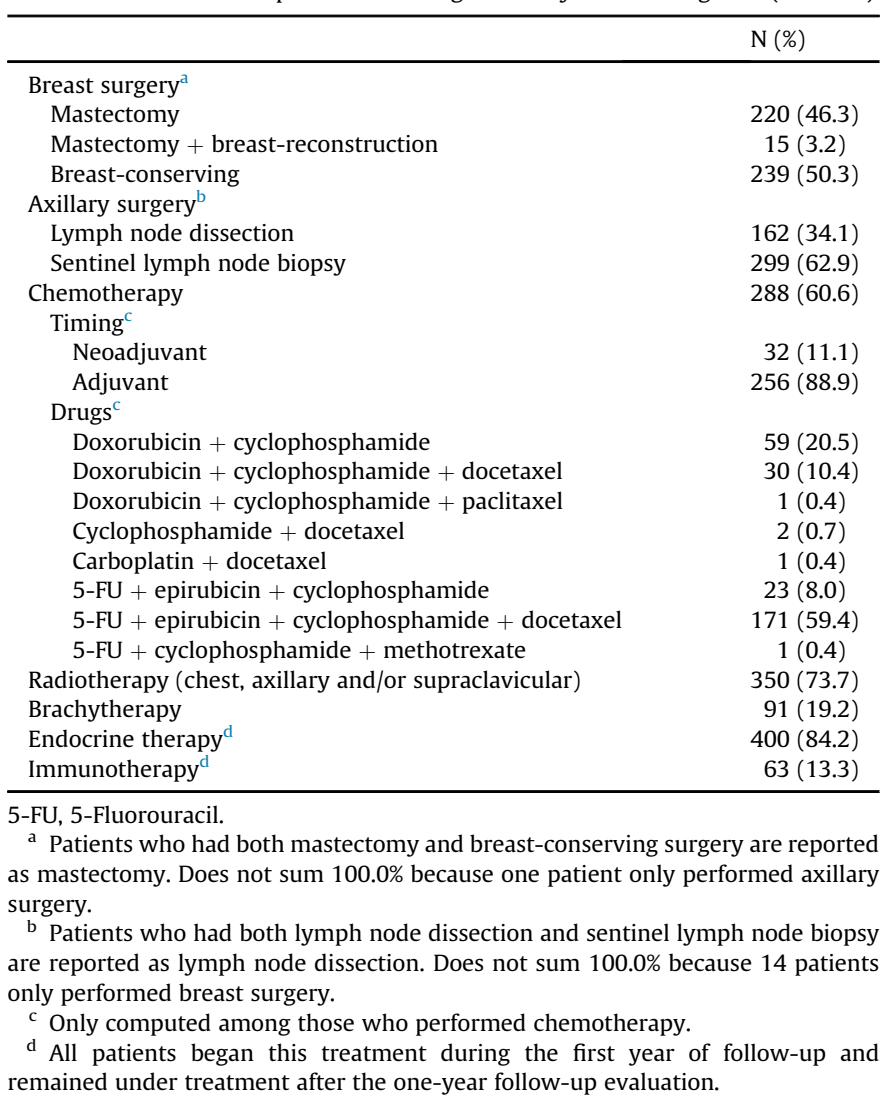

Between the first and the third year of follow-up, less than $2 \%$ of the patients underwent each of the following treatments: ALND, metastasectomy, radiotherapy or chemotherapy (Table 2).

Prevalence of neurological complications at one and three years of follow-up

Just over $40 \%$ of breast cancer patients had at least one cancerrelated neurological complication, at one $(42.7 \%$, 95\%Cl:

Table 2

Cancer treatments performed after the first year after diagnosis $(\mathrm{N}=475)$.

\begin{tabular}{ll}
\hline & $\mathrm{N}(\%)$ \\
\hline $\begin{array}{l}\text { Breast surgery } \\
\text { Breast-reconstruction }\end{array}$ & $26(5.5)$ \\
$\begin{array}{l}\text { Axillary surgery } \\
\text { Lymph node dissection }\end{array}$ & $1(0.2)$ \\
Hepatic metastasectomy & $1(0.2)$ \\
Cerebral metastasectomy & $1(0.2)$ \\
Chemotherapy & $9(1.9)^{\mathrm{a}}$ \\
Drugs & \\
$\quad$ Capecitabine & $3(33.3)$ \\
$\quad$ Docetaxel & $2(22.2)$ \\
$\quad$ Paclitaxel & $5(55.6)$ \\
$\quad$ Vinorelbine & $1(11.1)$ \\
$\quad$ Rituximab + cyclophosphamide + doxorubicin + vincristine & $1(11.1)^{\mathrm{c}}$ \\
Radiotherapy (chest, axillary, supraclavicular, & $7(1.5)$ \\
$\quad$ bone and/or cerebral) & \\
\hline
\end{tabular}

a Only one of these patients was not submitted to chemotherapy during the first year of follow-up.

b Only computed among those who performed chemotherapy. Does not sum $100.0 \%$ because some patients were submitted to more than one scheme after the first year after diagnosis.

${ }^{c}$ Patient submitted to chemotherapy due to a second primary cancer.
38.4-47.2) and three years of follow-up (41.7\%, 95\%CI: 37.3-46.2). More than half of the participants (54.7\%, 95\%CI: 50.2-59.2) presented at least one neurological complication, either at one- or at three-year follow-up evaluation.

As depicted in Fig. 1, between the first and the third year after breast cancer diagnosis, there was a non-statistically significant increase in the prevalence of NP, from $21.1 \%$ to $23.6 \%$ ( $p=0.225$ ), and a significant decrease in the proportion of women with phantom breast syndrome, from $17.1 \%$ to $10.7 \%$ ( $p<0.001$ ). The prevalence of cognitive impairment varied from $7.2 \%$ to $8.2 \%$ ( $p=0.466$ ), though $55.9 \%$ and $28.2 \%$ of those with cognitive impairment at one and three years, respectively, already presented this condition prior to treatment. The variation in the prevalence of cerebrovascular disease (from $0.6 \%$ to $1.5 \%$; $p=0.125$ ) and CIPN (from $14.1 \%$ to $12.6 \%$; $p=0.127$ ) was not statistically significant. Brain metastasis were identified in three patients $(0.6 \%, 95 \% \mathrm{CI}$ : $0.1-1.9)$ at the three-year evaluation.

The association between different characteristics of the patients and the presence of NP at one and three years after cancer diagnosis are reported in Table 3. At one year, older patients were less likely to present NP and those with cancer stage III/IV and those who underwent ALND presented higher odds of NP. At three years after cancer diagnosis only the patients submitted to chemotherapy had higher odds of NP. Those with NP at one year after cancer diagnosis had eight fold higher odds of NP at three years ( $\mathrm{OR}=8.20,95 \% \mathrm{CI}$ : 4.84-13.9, adjusted for all variables presented in Table 3).

The association between different characteristics of the patients and the presence of CIPN at one and three years after cancer diagnosis are reported in Table 4. Cancer stage III/IV and taxanebased chemotherapy were associated with a higher odds of CIPN, both at one and three years. Patients undergoing chemotherapy between the first and the third year had higher odds of CIPN at the three-year follow-up than those treated with chemotherapy only during the first year (OR $=11.7,95 \% \mathrm{CI}$ : 2.16-63.89, adjusted for all variables presented in Table 4).

Fig. 2 depicts the number of patients with the most common neurological complications, according to their presence at one and three years of follow-up. More than three quarters of the women with CIPN and more than half of those with NP remained affected by these conditions three years after cancer diagnosis. A total of $66.7 \%$ of the patients presenting cognitive impairment and $49.1 \%$ of those with NP at the three-year follow-up were not prevalent cases at the one-year evaluation.

Pain severity scores among patients with NP are presented in Fig. 3. Among those with NP in both moments there was a significant increase in the median score ( 2.4 vs. $3.2, \mathrm{p}<0.005)$. NP was less severe among women presenting this condition at the one-year evaluation only, than those with NP at the three-year follow-up only (median score: 1.2 vs. $2.5, \mathrm{p}<0.001$ ).

Among those with CIPN in both moments, there was a significant decrease in the median score of TNSc (5.0 vs. $4.0, \mathrm{p}=0.006$ ). CIPN had greater severity among women presenting this condition at the three-year evaluation only, than those with CIPN at the one-year follow-up only (median score: 10.0 vs. $1.5, \mathrm{p}<0.005$ ) (Fig. 4).

According to the CTCAE, in patients with CIPN at both moments of follow-up, almost all presented peripheral sensory neuropathy grade 1 or $2(100.0 \%$ at the one- and $98.1 \%$ at the three-year follow-up); motor symptoms (grade 1 to 3 ) were much less frequent (7.6\% at the one- and $15.1 \%$ at the three-year follow-up). Among women with CIPN at only the one-year or at the three-year follow-up evaluation, the prevalence of sensory neuropathy (grade 1 to 3 ) was $92.9 \%$ and $100.0 \%$, respectively, and the proportion of patients with motor neuropathy (grade 1 ) was $7.1 \%$ and $57.1 \%$, respectively. 


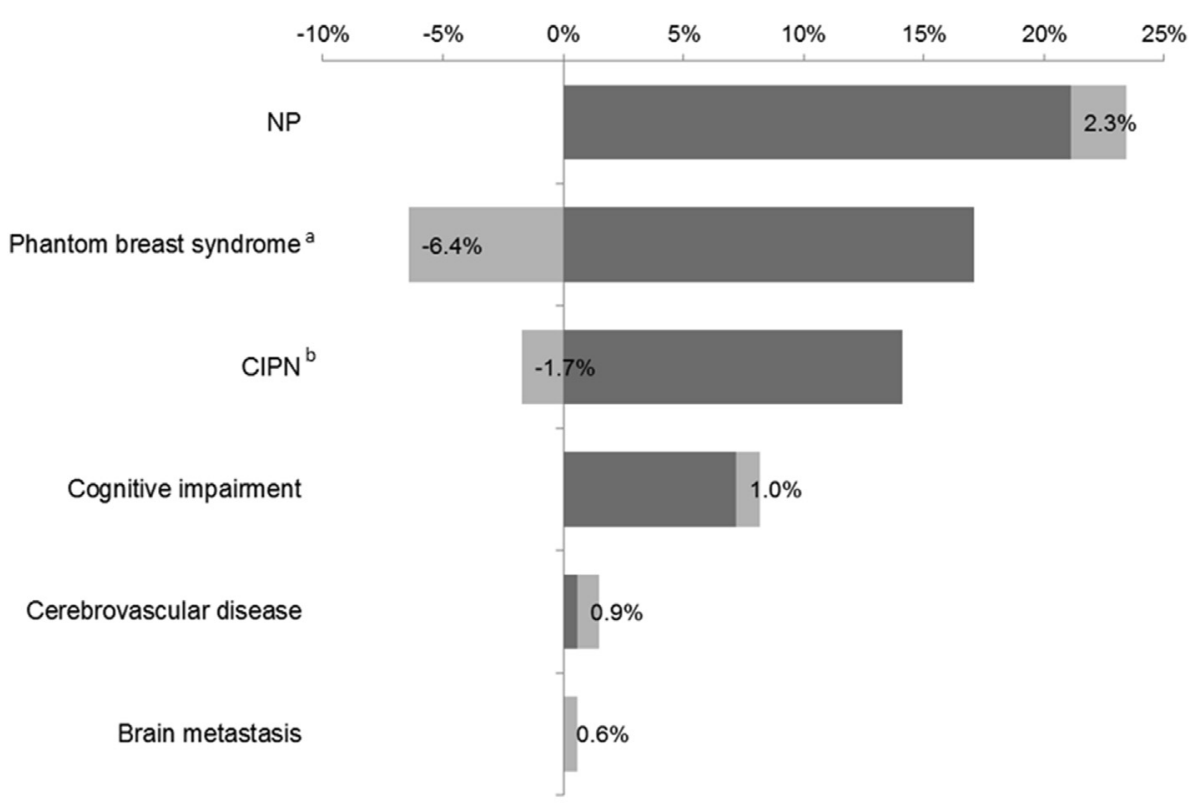

m Prevalence at one-yr $\quad$ Difference between the 1st and the 3rd $\mathrm{yr}$

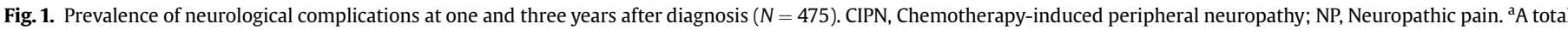

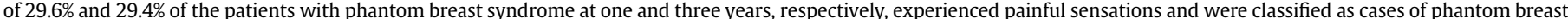

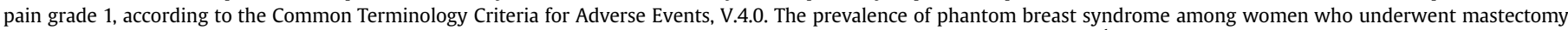

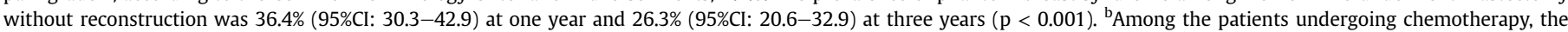
prevalence of CIPN was $23.3 \%$ (95\%CI: $18.7-28.5)$ and $20.5 \%(95 \% \mathrm{CI}: 16.2-25.5)$ at one and three years $(\mathrm{p}=0.074)$, respectively.

Table 3

Association between sociodemographic and clinical characteristics of the patients and NP at one and three years after cancer diagnosis $(\mathrm{N}=475)$.

\begin{tabular}{|c|c|c|c|c|}
\hline & \multicolumn{2}{|c|}{ Neuropathic pain at one year } & \multicolumn{2}{|c|}{ Neuropathic pain at three years } \\
\hline & Patients with NP [N (\%)] & Adjusted OR (95\%CI) & Patients with NP [N (\%)] & Adjusted OR (95\%CI) \\
\hline \multicolumn{5}{|l|}{ Age (years) } \\
\hline$\leq 55$ & $61(25.3)$ & 1 (ref.) & $64(26.6)$ & 1 (ref.) \\
\hline$>55$ & $39(16.7)$ & $0.59(0.38-0.93)$ & $48(20.5)$ & $0.71(0.47-1.09)$ \\
\hline \multicolumn{5}{|l|}{ Education (years) } \\
\hline$\leq 4$ & $40(20.0)$ & 1 (ref.) & $51(25.5)$ & 1 (ref.) \\
\hline $5-9$ & $29(21.5)$ & $0.86(0.48-1.53)^{c}$ & $28(20.7)$ & $0.62(0.35-1.08)^{c}$ \\
\hline$\geq 10$ & $31(22.1)$ & $0.87(0.49-1.55)^{c}$ & $33(25.6)$ & $0.71(0.41-1.24)^{\mathrm{c}}$ \\
\hline \multicolumn{5}{|c|}{ Cancer stage at baseline } \\
\hline $0 / \mathrm{I}$ & $40(15.6)$ & 1 (ref.) & $53(20.7)$ & 1 (ref.) \\
\hline II & $29(19.9)$ & $1.30(0.76-2.22)^{\mathrm{d}}$ & $36(24.7)$ & $1.27(0.78-2.07)^{\mathrm{d}}$ \\
\hline III/IV & $31(42.5)$ & $3.83(2.13-6.86)^{d}$ & $23(31.5)$ & $1.75(0.97-3.16)^{d}$ \\
\hline \multicolumn{5}{|c|}{ Breast surgery $^{\mathrm{a}}(\mathrm{N}=474)$} \\
\hline Breast-conserving & $39(16.3)$ & 1 (ref.) & $53(22.2)$ & 1 (ref.) \\
\hline Mastectomy & $61(26.0)$ & $1.33(0.82-2.17)^{\mathrm{e}}$ & $59(25.1)$ & $1.01(0.64-1.60)^{\mathrm{e}}$ \\
\hline \multicolumn{5}{|c|}{ Axillary surgery $^{\mathrm{b}}(\mathrm{N}=461)$} \\
\hline SLNB & $44(14.7)$ & 1 (ref.) & $58(19.5)$ & 1 (ref.) \\
\hline ALND & $56(34.6)$ & $2.75(1.34-5.63)^{\mathrm{e}}$ & $49(30.1)$ & $1.59(0.82-3.07)^{\mathrm{e}}$ \\
\hline \multicolumn{5}{|l|}{ Chemotherapy } \\
\hline No & $26(13.9)$ & 1 (ref.) & $30(16.1)$ & 1 (ref.) \\
\hline Yes & $74(25.7)$ & $1.47(0.80-2.70)^{\mathrm{e}}$ & $82(28.4)$ & $2.10(1.20-3.67)^{\mathrm{e}}$ \\
\hline \multicolumn{5}{|l|}{ Radiotherapy } \\
\hline No & $24(19.2)$ & 1 (ref.) & $24(19.4)$ & 1 (ref.) \\
\hline Yes & $76(21.7)$ & $0.72(0.32-1.59)^{\mathrm{f}}$ & $88(25.1)$ & $1.24(0.58-2.65)^{\mathrm{f}}$ \\
\hline \multicolumn{5}{|l|}{ Brachytherapy } \\
\hline No & $89(23.2)$ & 1 (ref.) & $91(23.7)$ & 1 (ref.) \\
\hline Yes & $11(12.1)$ & $0.53(0.24-1.15)^{\mathrm{f}}$ & $21(32.1)$ & $0.93(0.48-1.82)^{\mathrm{f}}$ \\
\hline
\end{tabular}

ALND, Axillary lymph node dissection; NP, Neuropathic pain; SLNB, Sentinel lymph node biopsy.

a Patients who had both mastectomy and breast-conserving surgery are reported as mastectomy; $\mathrm{N}<475$ because one patient only performed axillary surgery.

b Patients who had both ALND and SLNB are reported as ALND; $\mathrm{N}<475$ because 14 patients only performed breast surgery.

c Adjusted for age.

$\mathrm{d}$ Adjusted for age and education.

e Adjusted for age, education and cancer stage.

$\mathrm{f}$ Adjusted for age, education, cancer stage, breast and axillary surgery. 
Table 4

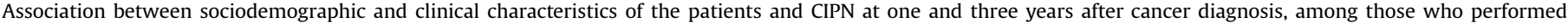
chemotherapy until the first $(\mathrm{N}=288)$ and until the third year of follow-up $(\mathrm{N}=289)$.

\begin{tabular}{|c|c|c|c|c|}
\hline & \multicolumn{2}{|l|}{ CIPN at one year } & \multicolumn{2}{|l|}{ CIPN at three years } \\
\hline & Patients with CIPN [N (\%)] & Adjusted OR (95\%CI) & Patients with CIPN [N (\%)] & Adjusted OR (95\%CI) \\
\hline \multicolumn{5}{|l|}{ Age (years) } \\
\hline$\leq 55$ & $39(22.7)$ & 1 (ref.) & $33(19.2)$ & 1 (ref.) \\
\hline$>55$ & $28(24.1)$ & $1.09(0.62-1.89)$ & $27(23.1)$ & $1.26(0.71-2.24)$ \\
\hline \multicolumn{5}{|c|}{ Education (years) } \\
\hline$\leq 4$ & $22(22.0)$ & 1 (ref.) & $21(20.8)$ & 1 (ref.) \\
\hline $5-9$ & $21(21.0)$ & $0.99(0.49-2.02)^{\mathrm{a}}$ & $17(17.0)$ & $0.87(0.41-1.83)^{\mathrm{a}}$ \\
\hline$\geq 10$ & $24(27.3)$ & $1.41(0.69-2.85)^{\mathrm{a}}$ & $22(25.0)$ & $1.42(0.69-2.93)^{\mathrm{a}}$ \\
\hline \multicolumn{5}{|c|}{ Cancer stage at baseline } \\
\hline $0 / \mathrm{I}$ & $12(13.2)$ & 1 (ref.) & $13(14.1)$ & 1 (ref.) \\
\hline II & $29(22.8)$ & $2.01(0.96-4.22)^{\mathrm{b}}$ & $22(17.3)$ & $1.34(0.63-2.86)^{\mathrm{b}}$ \\
\hline III/IV & $26(37.1)$ & $4.12(1.87-9.06)^{\mathrm{b}}$ & $25(35.7)$ & $3.73(1.70-8.14)^{b}$ \\
\hline \multicolumn{5}{|c|}{ 5-FU-based chemotherapy } \\
\hline No 5-FU & $15(16.1)$ & (ref.) & $16(17.0)$ & (ref.) \\
\hline 5-FU & $52(26.7)$ & $1.99(1.00-4.00)^{\mathrm{C}}$ & $44(22.6)$ & $1.65(0.83-3.30)^{\mathrm{c}}$ \\
\hline \multicolumn{5}{|c|}{ Taxane-based chemotherapy } \\
\hline No taxane & $1(1.20)$ & 1 (ref.) & $2(2.5)$ & 1 (ref.) \\
\hline Taxane & $66(32.2)$ & $34.59(4.57-261.96)^{c}$ & $58(27.9)$ & $14.76(3.31-65.79)^{\mathrm{c}}$ \\
\hline
\end{tabular}

CIPN, Chemotherapy-induced peripheral neuropathy; 5-FU, 5-Fluorouracil.

a Adjusted for age.

b Adjusted for age and education.

c Adjusted for age, education and cancer stage.

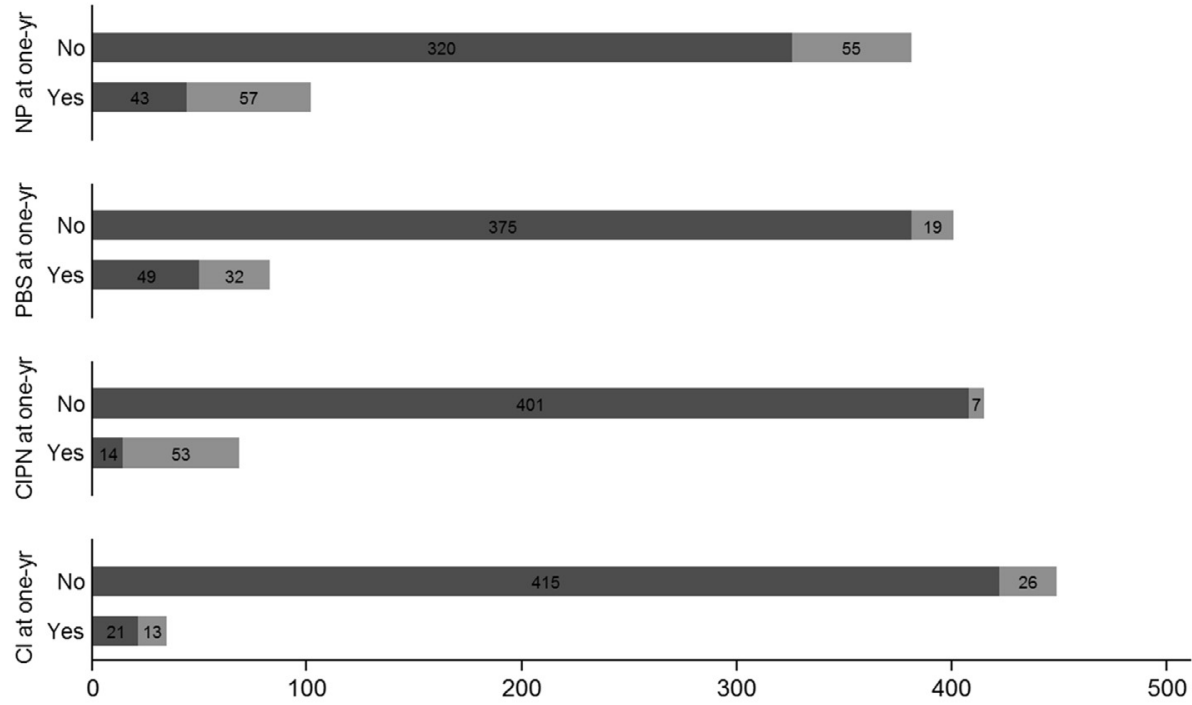

Presence of the neurological complication ar three-yrs No Yes

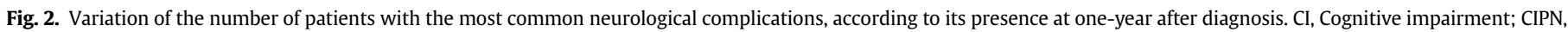
Chemotherapy-induced peripheral neuropathy; NP, Neuropathic pain; PBS, Phantom breast syndrome.

\section{Discussion}

Three years after a diagnosis of breast cancer, more than $40 \%$ of the patients presented at least one oncological-related neurological complication, largely due to conditions that were not observed one year after cancer diagnosis. During this period, there was an increase in the prevalence of NP, cognitive impairment, cerebrovascular disease and brain metastasis and a decrease in the proportion of patients with phantom breast syndrome and CIPN, though differences were not statistically significant except for phantom breast syndrome and brain metastasis. ALND was associated with NP at one year and chemotherapy with NP at three years. Taxane-based chemotherapy was strongly associated with prevalence of CIPN at one and three years.

NP was the most frequent treatment-related complication, both one and three years after cancer diagnosis. Two recent studies addressing the frequency of NP in breast cancer patients reported prevalence estimates close to those observed in our study $[16,17]$; in a prospective study of women who underwent surgery for primary breast cancer, Bruce et al. reported a prevalence of $24 \%$ of predominantly NP at nine months, according to the SelfAdministered Leeds Assessment of Neuropathic Symptoms and 


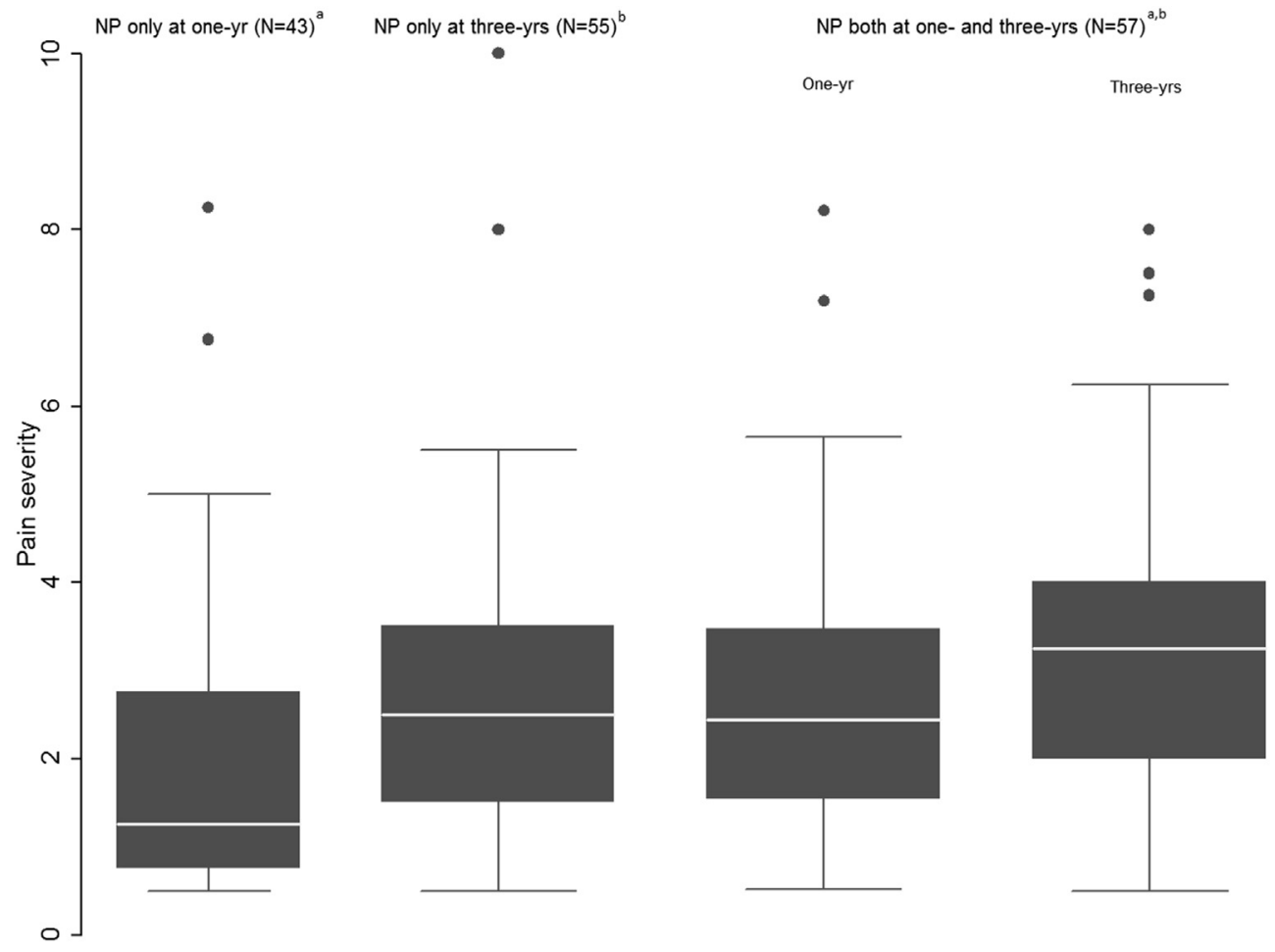

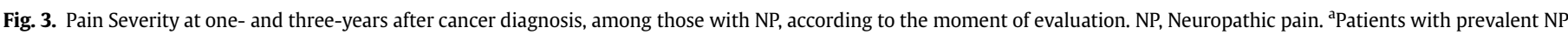

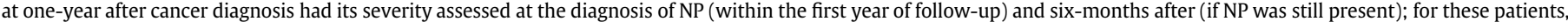

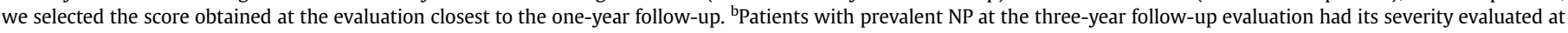
that moment.
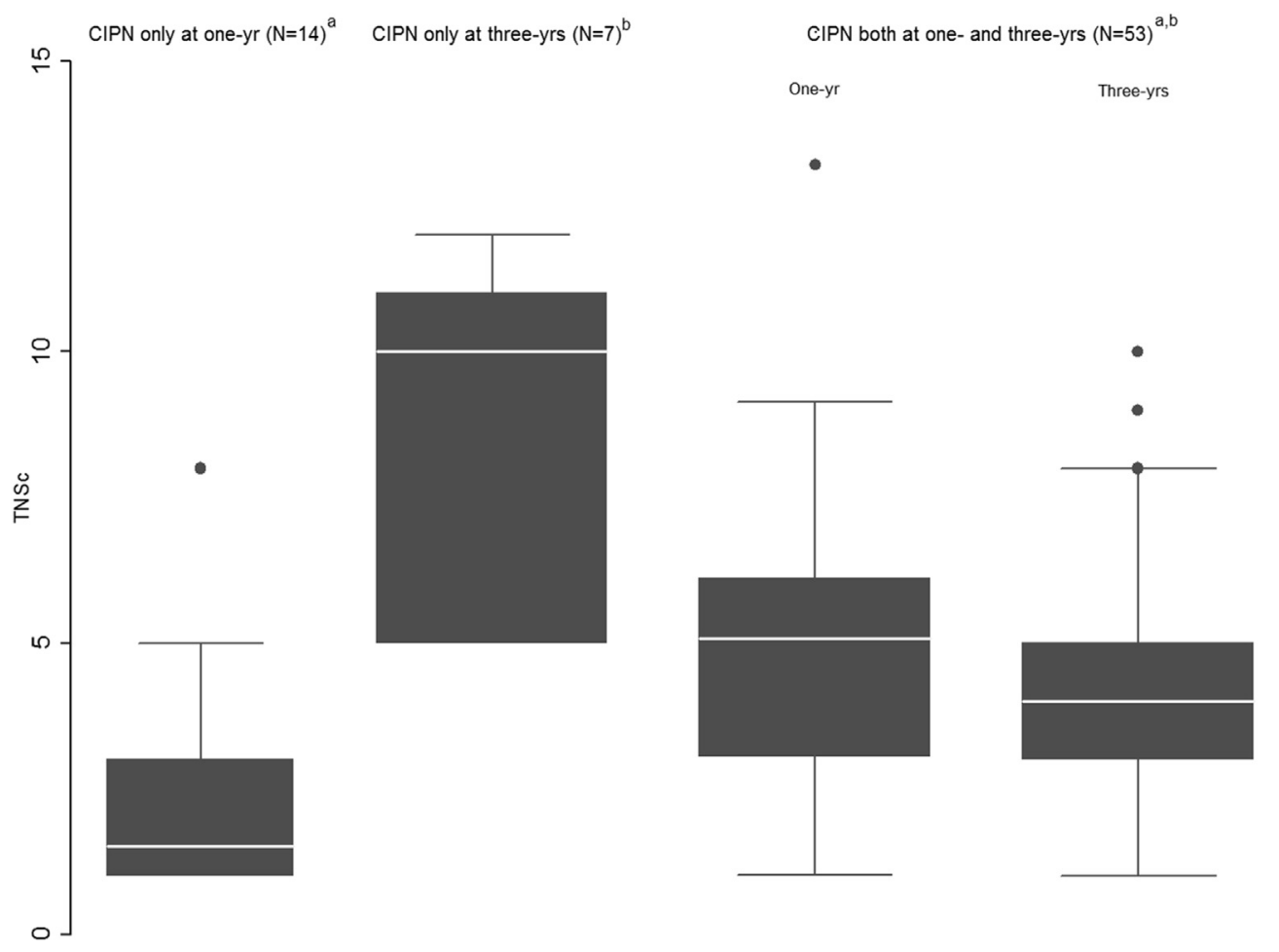

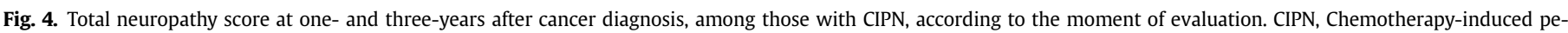

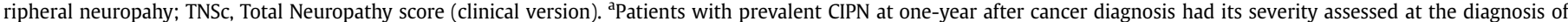

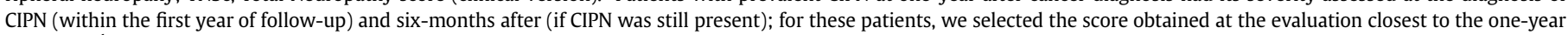

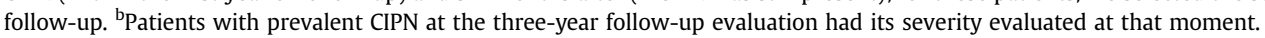


Signs (S-LANSS) or the Neuropathic pain questionnaire (DN4) [16]. In a retrospective study including women treated between two to six years before, Bredal et al. described the presence of symptoms and signs of NP in $26 \%$ of the participants, according to the S-LANSS [17].

Even though NP is a frequently recognized complication of surgical and adjuvant treatments for breast cancer [15,18], there is scarce information regarding how it evolves in the long term in the same patients. The fact that signs and symptoms that characterize NP occur as a dynamic spectrum that is unstable over time $[19,20]$ could, at least in part, explain our findings of an increase in its prevalence during follow-up. Among breast cancer patients, damage of the intercostobrachial nerve, which can occur with ALND, has been considered the most common source of NP [15] and patients submitted to chemotherapy may develop NP secondary to CIPN [18]; our results are in agreement with these findings.

Our study yielded a prevalence of phantom breast syndrome similar to those found by previous authors reporting that between $15 \%$ and $45 \%$ of those submitted to mastectomy were diagnosed with this complication [21-23]. Recently, Medina et al., reported a decrease in the prevalence from $45 \%$ at six weeks to $18 \%$ at two years after surgery [21], which is a accordance to our findings of a decrease over time.

Despite direct comparisons being difficult due to the heterogeneous chemotherapeutic schemes performed by patients and the lack of uniformity in CIPN assessment methods, our observations of a decrease in the prevalence of CIPN between the first and the third year after diagnosis are consistent with previous studies [24,25]. A systematic review assessing the prevalence of CIPN over time yielded prevalence estimates of $60.8 \%$ in the first month after chemotherapy, $60.0 \%$ at three months and $30.0 \%$ at six months or more [25]. In a more recent study, Eckhoff et al. reported a CIPN prevalence of $16.2 \%$ and $13.6 \%$ among breast cancer survivors who underwent docetaxel based-regimens, 1-1.5 years and 2.0-3.2 years after treatment, respectively [24]. The low number of patients with CIPN diagnosis at only three years reflects the scarce number of women re-submitted to chemotherapy due to cancer recurrence and its higher severity when compared to those diagnosed at oneyear may reflect the use of more neurotoxic drugs [26]. Also, our finding of a higher odds of CIPN in those who underwent taxanebased chemotherapy is in accordance with previous reports suggesting that both docetaxel and paclitaxel could be an important source of peripheral neuropathy among those undergoing chemotherapy for breast cancer $[18,26]$.

Cognitive impairment has been studied among breast cancer patients, especially in those submitted to chemotherapy [27-29]. Results from cross-sectional studies suggest a decrease in cognitive function after chemotherapy but prospective designs show an improvement in cognition over time after treatment [27-29]. However, the former did not take into account the baseline cognitive status of patients and the latter may be explained by learning effects due to evaluations using the same instrument at different moments. In our study, the long period between the oneand the three-year follow-up evaluations contributes to overcome learning effects and may explain the contrast with previous results.

Cerebrovascular disease and brain metastasis have been found in less than $2 \%$ of the participants in our study. The latter has been describes as the most common direct form of nervous system involvement by cancer, estimated to affect around $5 \%$ of breast cancer patients, up to five years after diagnosis [30]; the shorter follow-up of our cohort and the fact that those not proposed for surgery were excluded, are probably contributing to our observed prevalence.

Our study contributes to a comprehensive characterization of the burden of neurological conditions of breast cancer in the long term. Its major methodological strengths are the prospective design with a systematic evaluation of all patients before treatment, one year and three years after breast cancer diagnosis, the nearly complete follow-up of the patients and the use of standardized instruments to evaluate cognitive function, and to quantify CIPN and NP severity. However, some limitations need to be addressed. We excluded those not submitted to surgical treatment, which may limit the generalization to patients with more advanced disease, where direct complications of breast cancer are expected to be more frequent. Finally, most of the neurological complications had only clinical diagnosis, which limits the accuracy of the information regarding these disorders. However, our results are expected to reflect findings in usual clinical practice, since all patients were treated according to the usual practice of a major oncological hospital.

In conclusion, our study shows that neurological complications are frequent even three years after cancer diagnosis and highlights the role of NP as a major contributor to the burden of these conditions among survivors.

\section{Ethical approval}

The study was approved by the Ethics Committee of the Portuguese Institute of Oncology of Porto (Ref. CES 406/011 and Ref. 99/ 014).

\section{Conflict of interest statement}

None declared.

\section{Acknowledgments}

The work of FF was supported by the "Fundação para a Ciência e a Tecnologia" (SFRH/BD/92630/2013), data management activities up to the first year of follow-up were supported by the Chair on Pain Medicine of the Faculty of Medicine, University of Porto and by the Grünenthal Foundation - Portugal and the three-year followup was supported by "Fundo Europeu de Desenvolvimento Regional" (FEDER- 016867) and by the "Fundação para a Ciência e a Tecnologia" (PTDC/DTP-EPI/7183/2014).

Epidemiology Research Unit (EPIUnit) is funded by the "Fundação para a Ciência e a Tecnologia" (UID/DTP/04750/2013).

\section{References}

[1] Ferlay J, Soerjomataram I, Ervik M, Dikshit R, Eser S, Mathers C, et al. GLOBOCAN 2012 v1.0, cancer incidence and mortality worldwide: IARC CancerBase No. 11 [Internet]. Lyon, France: International Agency for Research on Cancer; 2013. Available from: http://globocan.iarc.fr (Accessed on 15 February 2016).

[2] Amaro J, Severo M, Vilela S, Fonseca S, Fontes F, La Vecchia C, et al. Patterns of breast cancer mortality trends in Europe. Breast 2013:22(3):244-53.

[3] Allemani C, Weir HK, Carreira H, Harewood R, Spika D, Wang XS, et al. Global surveillance of cancer survival 1995-2009: analysis of individual data for 25,676,887 patients from 279 population-based registries in 67 countries (CONCORD-2). Lancet 2015:385(9972):977-1010.

[4] Khasraw M, Posner JB. Neurological complications of systemic cancer. Lancet Neurol 2010;9(12):1214-27.

[5] Giglio P, Gilbert MR. Neurologic complications of cancer and its treatment. Curr Oncol Rep 2010:12(1):50-9.

[6] Pereira S, Fontes F, Sonin T, Dias T, Fragoso M, Castro-Lopes JM, et al. Neurological complications of breast cancer: a prospective cohort study. Breast 2015:24(5):582-7.

[7] Pereira S, Fontes F, Sonin T, Dias T, Fragoso M, Castro-Lopes JM, et al. Neurological complications of breast cancer: study protocol of a prospective cohort study. BMJ Open 2014:4(10):e006301.

[8] Freitas S, Simoes MR, Alves L, Santana I. Montreal cognitive assessment (MoCA): normative study for the Portuguese population. J Clin Exp Neuropsychol 2011;33(9):989-96. 
[9] Freitas S, Simoes MR, Martins C, Vilar M, Santana I. Adaptation studies of the montreal cognitive assessment (MoCA) to the Portuguese population. Aval Psicol 2010;9(3):345-57.

[10] Edge S, Byrd DR, Compton CC, Fritz AG, Greene FL, Trotti A. AJCC cancer staging manual. 7th ed. New York: Springer; 2010.

[11] Cornblath DR, Chaudhry V, Carter K, Lee D, Seysedadr M, Miernicki M, et al. Total neuropathy score: validation and reliability study. Neurology 1999;53(8):1660-4.

[12] CTCAE. Cancer Therapy Evaluation Program, Common terminology criteria for adverse events, version 4.0. DCTD, NCI, NIH, DHHS. 2010. http://ctep.cancer. gov/ (Accessed on 1 January 2012).

[13] Haanpaa M, Attal N, Backonja M, Baron R, Bennett M, Bouhassira D, et al. NeuPSIG guidelines on neuropathic pain assessment. Pain 2011;152(1): $14-27$.

[14] Cleeland CS, Ryan KM. Pain assessment: global use of the brief pain inventory. Ann Acad Med Singap 1994:23(2):129-38.

[15] Jung BF, Ahrendt GM, Oaklander AL, Dworkin RH. Neuropathic pain following breast cancer surgery: proposed classification and research update. Pain 2003; 104(1-2):1-13.

[16] Bruce J, Thornton AJ, Powell R, Johnston M, Wells M, Heys SD, et al. Psychological, surgical, and sociodemographic predictors of pain outcomes after breast cancer surgery: a population-based cohort study. Pain 2014;155(2): 232-43.

[17] Schou Bredal I, Smeby NA, Ottesen S, Warncke T, Schlichting E. Chronic pain in breast cancer survivors: comparison of psychosocial, surgical, and medical characteristics between survivors with and without pain. J Pain Symptom Manage 2014;48(5):852-62.

[18] Jung BF, Herrmann D, Griggs J, Oaklander AL, Dworkin RH. Neuropathic pain associated with non-surgical treatment of breast cancer. Pain 2005;118(1-2): $10-4$.

[19] Bennett MI, Smith BH, Torrance N, Lee AJ. Can pain can be more or less neuropathic? Comparison of symptom assessment tools with ratings of certainty by clinicians. Pain 2006;122(3):289-94.
[20] Attal N, Bouhassira D. Can pain be more or less neuropathic? Pain 2004;110(3):510-1.

[21] Medina Jde M, Fabro EA, Amaral e Silva B, Thuler LC, Bergmann A. Frequency and associated factors of phantom breast syndrome in women submitted to mastectomy for breast cancer. Rev Bras Ginecol Obstet 2015;37(9):397-401.

[22] Ahmed A, Bhatnagar S, Rana SP, Ahmad SM, Joshi S, Mishra S. Prevalence of phantom breast pain and sensation among postmastectomy patients suffering from breast cancer: a prospective study. Pain Pract $2014 ; 14(2)$ E17-28.

[23] Hansen DM, Kehlet H, Gartner R. Phantom breast sensations are frequent after mastectomy. Dan Med Bull 2011;58(4):A4259.

[24] Eckhoff L, Knoop A, Jensen MB, Ewertz M. Persistence of docetaxel-induced neuropathy and impact on quality of life among breast cancer survivors. Eur J Cancer 2015;51(3):292-300

[25] Seretny M, Currie GL, Sena ES, Ramnarine S, Grant R, MacLeod MR, et al. Incidence, prevalence, and predictors of chemotherapy-induced periphera neuropathy: a systematic review and meta-analysis. Pain 2014;155(12): $2461-70$.

[26] Miltenburg NC, Boogerd W. Chemotherapy-induced neuropathy: a comprehensive survey. Cancer Treat Rev 2014:40(7):872-82.

[27] Stewart A, Bielajew C, Collins B, Parkinson M, Tomiak E. A meta-analysis of the neuropsychological effects of adjuvant chemotherapy treatment in women treated for breast cancer. Clin Neuropsychol 2006;20(1):76-89.

[28] Falleti MG, Sanfilippo A, Maruff P, Weih L, Phillips KA. The nature and severity of cognitive impairment associated with adjuvant chemotherapy in women with breast cancer: a meta-analysis of the current literature. Brain Cogn 2005;59(1):60-70.

[29] Ono M, Ogilvie JM, Wilson JS, Green HJ, Chambers SK, Ownsworth T, et al A meta-analysis of cognitive impairment and decline associated with adjuvant chemotherapy in women with breast cancer. Front Oncol 2015:5:59.

[30] Dawood S, Lei X, Litton JK, Buchholz TA, Hortobagyi GN, GonzalezAngulo AM. Incidence of brain metastases as a first site of recurrence among women with triple receptor-negative breast cancer. Cancer 2012;118(19): 4652-9. 